\title{
Test Bench Design of The Micro Turbine Engine
}

\author{
Lv Qiongying \\ College of Mechanical and Electric Engineering \\ Changchun University of Science and Technology \\ Changchun, Jilin, China \\ e-mail: 13578868098@126.com
}

\author{
Quan Yazhou \\ College of Mechanical and Electric Engineering \\ Changchun University of Science and Technology \\ Changchun, Jilin, China \\ e-mail: quanyazhou@sina.com
}

\begin{abstract}
In view of the existing micro turbine engine (MTE) design a test bench, which can measurement the key performance parameters. When the micro turbine engine is in operation, we can measurement the exhaust temperature, the vibration, rotate speed of the engine, also the dynamic pressure and static pressure of the exhaust nozzle. This article also introduces the installation method of various measuring sensors. According to characteristic of thrust measurement using CREO based on the piezoelectric force sensor design a six degrees of freedom thrust measuring platform, in the case of normal operation of the micro turbine engine can sense the condition of the thrust in all directions, and then according to the characteristics of the thrust measurement platform confirm the calibration principle. And then based on ANSYS make a modal analysis for the whole thrust measuring bench, get the inherent frequency.
\end{abstract}

Keywords-Microm Turbine Engine;Thrust Measurement; Test bench;Structural Design; Modal Analysis

\section{INTRODUCTION}

Engine test bench is the basis experiment and scientific research, is the guarantee of increase and improve the quality of the engine. Experimental research on the micro turbine engine can get the technical data which theoretical calculation can't get, it can verify and perfect with the theoretical calculation method and showed the real engine performance. When the performance parameters confirmed from the experiment can't meet the technical requirements, you can change or adjust the technical performance parameters of the engine in real time. Through the experiment to verification the finally determine product design direction, technical parameters and comprehensive implementation details in the future. Measured engine requires an effective test method to obtain accurate test results and parameters, and then design an engine which has best performance and the most reliable. The rapid development of micro turbine engine test platform and test technology research also must satisfy the above requirements, therefore, high technical content of the test platform and test technology is indispensable.

\section{RESEARCH STATUS}

At present, many research teams, both at home and abroad are actively do the research and development work about overall performance test experiment of MTE. The Karlsruhe Institute of Technology tested a kind of micro turbine engine which designed thrust was only $30 \mathrm{~N}$ manufactured by Schneider-Sanches. Then carry out the engine performance test and data collection analysis ${ }^{[1]}$. Catholic University of Louvain designed and set up a complete set of the micro engine test bench which detailed testing the parameters of the MTE ${ }^{[2]}$. National Tsing Hua University in Taiwan of China carried out the dynamic and steady-state performance and components characteristics research of the whole machine experiments and then created a set of micro turbine engine design and test method $^{[3-4]}$. NASALewis researched a kind of micro thrust test bench which can thrust stand for pulsed plasma thrusters [5]. MSFC's Propulsion Research and Development Laboratory recently developed the Variable Amplitude Hanging pendulum with Extended Range (VAHPER) thrust stand, which has proven over a range of thrust levels from 1 milliNewton to 1 Newton to have very good measurement resolution of 0.1 milliNewtons. This thrust stand will be used to measure the thrust produced by E-IMPAcT to independently confirm and extend the measurements previously acquired in the Princeton laboratories ${ }^{[6]}$.From 2001 the Nanjing University of Aeronautics and Astronautics had conducted extensive research and experiment for micro turbine engine, at present they have been successfully designed and manufactured out of the four types of micro turbojet engine principle prototype ${ }^{[7-8]}$, and has established a relatively complete miniature experiment platform and micro combustion experiment platform.

\section{MEASUREMENT METHOD AND SENSOR SELECTION}

\section{A. Exhaust Temperature Measurement}

In order to measuring the high temperature gas we choose the infrared thermometer was the CellaTemp PA which made by KELLER, Germany. The measurement principle is shown in Fig .1. The CellaTemp PA Pyrometer Series is based on a modular concept consisting of the following components: optics, sensor and signal processing, data output and target sighting. The optical system comes with one of five available lenses. Select the one most suitable, depending on the target size and measuring distance of your application. The pyrometer is infinitely adjustable to ensure superior precision across a wide focusing range. CellaTemp PA's signal processing is based on the latest DC technology and does not involve any moving parts, thus ensuring maintenance free, non- 
wearing operation. Signal conditioning combined with high resolution analogue-to-digital conversion enables a wide measuring span. The temperature resolution remains uniformly high across the entire measuring range. For measurement data output, Cella Temp PA features 2 analogue outputs, a USB interface and a networkcompatible RS 485 interface as well as two switching outputs. Three target sighting systems are available to facilitate focusing, alignment and indication of spot size: through-the-lens sighting, laser spot light or an integrated video camera. The individual components and subassemblies can be combined in different ways, yielding 24 instrument variants.

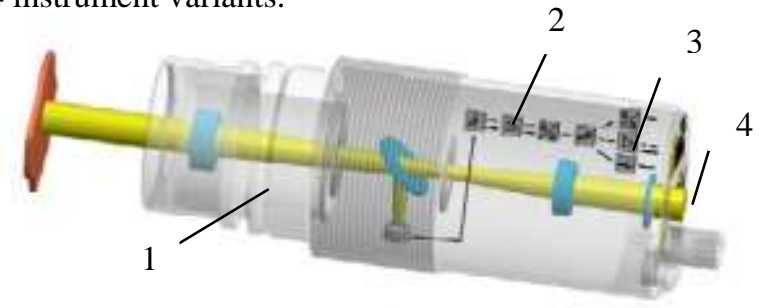

Figure 1. Measuring principle diagram

1-optical system 2-signal processing 3-data output 4-target sighting

\section{B. Rotate Speed Measurement}

The volume of measured micro turbine engine is small, we cannot use the contact method to measure the rotate speed, so this paper adopts eddy current sensor to measure the rotate speed. The method is to milling two keyway of symmetrical on the rotor shaft. As shown in Fig .2.The probe of eddy current sensor is facing the keyway. Because of the two keyway when the axis of rotation rotating, the eddy current sensor can outputs periodic continuous variation of the pulse signal, and then statistics on the number pulse signal. The speed of the rotor can be calculated. The eddy current sensor is KD2446 manufactured by KAMAN, America. This king of sensor's frequency response can up to $10 \mathrm{KHz}$. Theoretical rotating speed of micro turbine engine is $155000 \mathrm{rpm}$, so it can satisfy the measurement requirements.

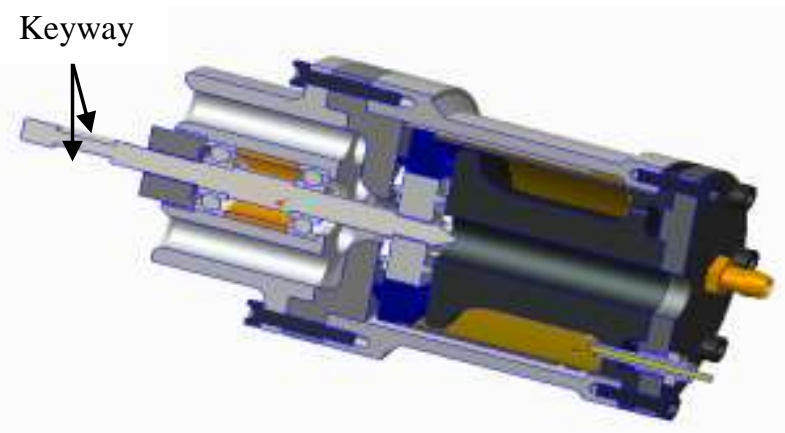

Figure 2. The method of rotate speed measurement

\section{Vibration Measurement}

We will use contact measurement to measure the vibration of micro turbine engine. But the vibration sensor should not be affecting the whole test bench. So we choose 8002 made by Kister. Type $8002 \mathrm{~K}$ is a high precision accelerometer for shock and vibration measurements in laboratory applications. Its excellent performance is derived from an ultra-stable crystalline quartz sensing element. The weight of the sensor is only $20 \mathrm{~g}$. Mounting is accomplished by inserting a Type 8402 stud into 10-32 UNF threaded hole in the object to be measured. Orthogonal measurements in three principal axes can be accomplished by utilizing a Type 8502 triaxial mounting cube.

\section{Static And Dynamic Pressure Measurement}

Pressure measurements during engine testing are taken using two probes - one static and one dynamic. The static probe is open to the inside of the engine nozzle, normal to the exhaust flow and near the outlet. The dynamic pressure sensor is on a track that enables position control during testing. While the engine is running, the dynamic probe is swept across the midline of the engine nozzle perpendicular to the exhaust flow. The dynamic pitot probe required a sufficiently rigid pitot tube to avoid the extreme velocities and temperatures it encountered during testing. Probe tip will feel the pressure signal transmitted to the scanner, then the scanner's internal sensors and signal processing systems will pass over the pressure signal into a digital signal. Pressure data is output over the Ethernet interface supporting both TCP and UDP protocols. The type of scanner is Net scanner Model 98RK which manufactured by Netscan.

\section{THE METHOD OF THRUST MEASUREMENT}

\section{A. 6-Degree of Freedom Static Thrust Test Bench}

Micro turbine engine thrust measurement system should have enough stiffness, high natural frequency, high sensitivity and good system stability. The test bench shall ensure that the measurement error is small, the thrust measurement accuracy. In order to meet the above requirements, the piezoelectric force transducer is a better choice. The main material of piezoelectric force transducer is piezoelectric quartz. Compared with other forms of force sensor it has advantage of high rigidity, high natural frequency, and compact structure. The overall structure of the thrust measurement system is shown in Fig .3

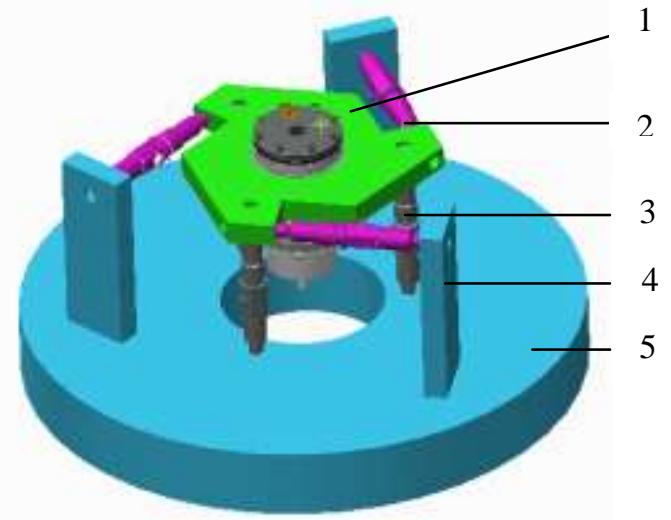

Figure 3. The overall design scheme of test bench

1- Fixed holder 2- Lateral force sensor 3- Longitudinal force sensor 4brace 5-bace

The thrust measurement platform mainly has four parts. The jet engine installed on the fixed holder. Three 
horizontal sensors and three longitudinal sensors were installed on the fixed holder. There are two thread hole of M3 which on both ends of the piezoelectric force transducer. It can fix the sensor. The total length of the sensor is $64 \mathrm{~mm}$, and installed directly between fixed holder and base. Three horizontal sensors into a $120^{\circ}$ Angle to each other and three longitudinal sensors into a $120^{\circ}$ Angle to each other too.

When the micro turbine engine is running the thrust measuring system can measurement the six thrust in space simultaneous. The jet engine is mounted with exhaust gases exiting upward and axial thrust acting downward, avoiding ground effects. Six load cells provide six degrees of freedom when characterizing engine thrust. Six sensors can force decomposition and then output charge ${ }^{[9]}$. Through the charge amplifier to processing signal, then will enter the final result to the compute.

As mentioned earlier, the thrust stand was designed so the engine exhausts plume exits vertically, and the thrust acts downward into the test cart. The load cells are divided into axial $[A a, B a$, and $C a]$ and lateral $[A l, B l$, and $C l]$ groups based on the orientation of their sensing axes. The coordinate system for the thrust stand, pictured in Fig .4.1, is defined with the $\mathrm{z}$-axis acting vertically upward along the axial centerline of the engine. Thus, the thrust acts in the negative $\mathrm{z}$-direction. The $\mathrm{y}$-axis runs perpendicular to and intersects with the longitudinal axis of the axial load cell, $A a$. The x-axis completes the system. Apositive rotational moment about the $y$-axis is equivalent to a positive pitching moment on the vehicle; a positive moment about the $\mathrm{z}$-axis is equivalent to a positive yawing moment on the vehicle; and a positive moment about the $\mathrm{x}$-axis is equivalent to a positive rolling moment on the vehicle. The origin of the coordinate system is centered in the plane of the engine mounts, and lies along the sensing axes of the lateral load cells ${ }^{[10]}$.

\section{B. Thrust measurement bench natural frequency analysis}

We should be validated whether the engine thrust test system meets the test requirements of thrust when in the design stage of micro turbine engine thrust test systems. The thrust of micro turbine engine changed quickly when it in testing process, so the thrust of the engine test system require a higher natural frequency. We will use finite element analysis software to do the modal analysis and simulation of thrust measurement test bench after the design completed. Then get the natural frequency and the vibration mode. It shows in Fig .4. It is the first six modal analysis results.

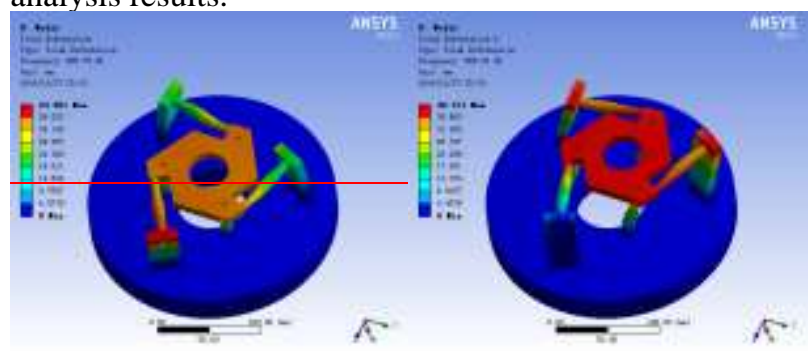

(1) The first modal

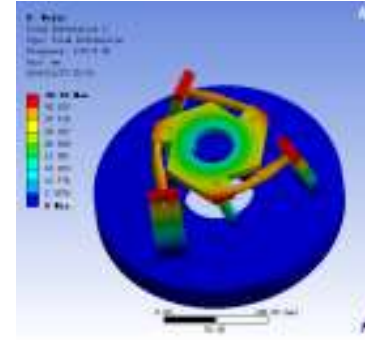

(3) The third modal

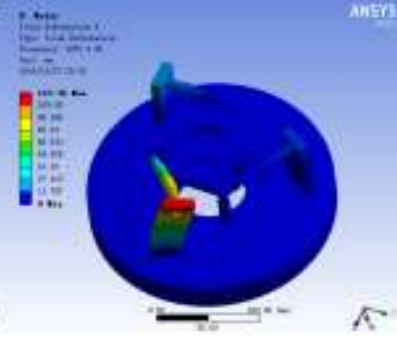

(4) The fourth modal

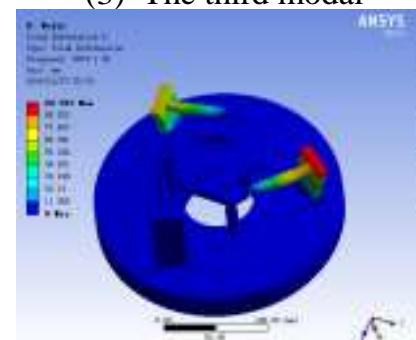

(5) The fifth modal

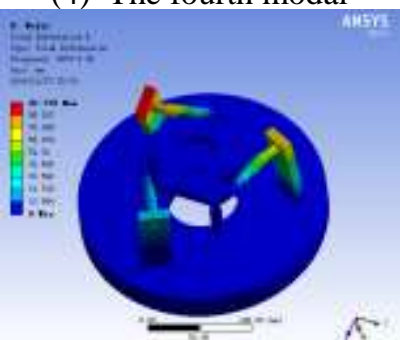

(6) The sixth modal
Figure 4. Modal analysis results

From the modal analysis results of test bench we can see the natural frequency is similar about first model and second model. The frequency is $865.75 \mathrm{~Hz}$ and $866.91 \mathrm{~Hz}$.And the fourth fifth sixth model is 3072.4 074.1 and $3074.5 \mathrm{~Hz}$. Theoretical rotating speed of micro turbine engine is $155000 \mathrm{rpm}$, so the maximum frequency of the engine is $2583 \mathrm{~Hz}$. Although the natural frequency of the former 2 modals is low, but considering the rotating speed of the engine is faster. So the resonate probability between engine and test bench is very low, can be neglected. The $2583 \mathrm{~Hz}$ is less than the natural frequency of test bench, so it can meet the requirements of frequency.

\section{Jet Engine Thrust Stand CALIBRATION PROCEDURE}

Because of the geometric complexity of the test stand, the entire test stand was calibrated for output forces and moments as a function of the 6 load cell readings ${ }^{[11]}$. For this calibration process, the test stand is mathematically modeled as a linear perturbation model of the form.

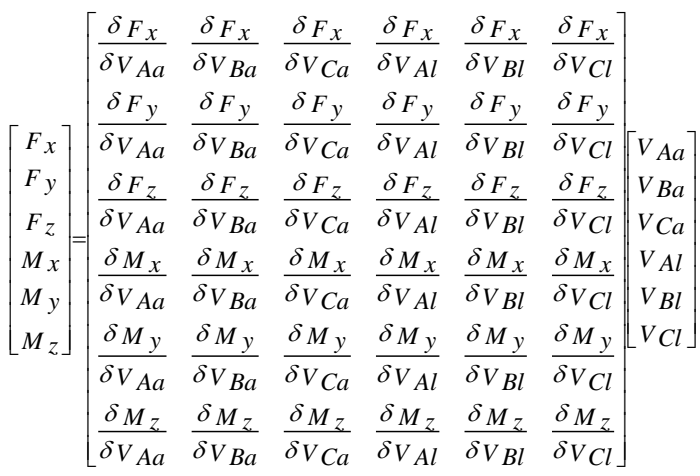

In Eq. (1) the vector [Fx, Fy, Fz, Mx, My, Mz] contains the desired forces and moments to be calculated when the thrust stand is loaded. The vector [VAa, VBa, $\mathrm{VCa}, \mathrm{Val}, \mathrm{VB} 1, \mathrm{VCl}]$ contains the output voltages from the six axial and lateral load cells as pictured in Fig .28. The linear system of Eq. (1) assumes that the voltage readings for the 6 load sensors have been adjusted so that zero-load on the thrust stand produces zero load cell output ${ }^{[12]}$. The 
36 elements of the Jacobian matrix define the externally applied forces and moments in terms of the sensed (and zeroed) load cell outputs. The calibration procedure reverses the process with multiple known external forces and moments applied to the thrust stand, and the corresponding sensor reading are logged. Assuming a set of $\mathrm{N}$ calibration inputs,

$$
\stackrel{\circ}{F}=\left\{\left[\begin{array}{c}
F_{x} \\
F_{y} \\
F_{z} \\
M_{x} \\
M_{y} \\
M_{z}
\end{array}\right]_{1}\left[\begin{array}{c}
F_{x} \\
F_{y} \\
F_{z} \\
M_{x} \\
M_{y} \\
M_{z}
\end{array}\right]_{2}\left[\begin{array}{l}
F_{x} \\
F_{y} \\
F_{z} \\
M_{x} \\
M_{z}
\end{array}\right]_{n}\right\}
$$

and a corresponding set of sensor outputs

$$
\stackrel{\circ}{V}=\left\{\left[\begin{array}{c}
V_{A a} \\
V_{B a} \\
V_{C a} \\
V_{A l} \\
V_{A l} \\
V_{A l}
\end{array}\right]_{1}\left[\begin{array}{c}
V_{A a} \\
V_{B a} \\
V_{C a} \\
V_{A l} \\
V_{A l} \\
V_{A l}
\end{array}\right]_{2}\left[\begin{array}{c}
V_{A a} \\
V_{B a} \\
V_{C a} \\
V_{A l} \\
V_{A l} \\
V_{A l}
\end{array}\right]_{n}\right\}
$$

Eq. (1) can be written as the $6 \times N$ dimensioned matrix equation

$$
\stackrel{\circ}{F}=J \stackrel{\circ}{V}
$$

In Eq. (4) $\mathrm{J}$ is the Jacobian matrix from Eq. (1). Postmultiplying both sides of Eq. (4) by $\stackrel{\circ}{V}^{T}$ produces the $6 \times$ 6 dimensioned system

$$
\stackrel{\circ}{F} \stackrel{\circ}{V}^{T}=J\left(\stackrel{\circ}{V} \stackrel{\circ}{V}^{T}\right)
$$

Post multiplying both sides by $\left(\stackrel{\circ}{V} \stackrel{\circ}{V}^{T}\right)^{-1}$ gives the least squares estimate for the Jacobian matrix

$$
y=\stackrel{\circ}{F} \stackrel{\circ}{V}^{T}\left(\stackrel{\circ}{V} \stackrel{\circ}{V}^{T}\right)^{-1}
$$

Now given an arbitrary set of load cell readings (zeroed for the no load case), the force and moment outputs from the thrust stand can be calculated as

$$
\left[\begin{array}{c}
F_{x} \\
F_{y} \\
F_{z} \\
M_{x} \\
M_{y} \\
M_{z}
\end{array}\right]=y\left[\begin{array}{c}
V_{A a} \\
V_{B a} \\
V_{C a} \\
V_{A l} \\
V_{A l} \\
V_{A l}
\end{array}\right]
$$

Calibration loads and moments are applied to the test stand using known calibration weights. Three axial and three lateral loads are applied at any one time, and the resulting forces and moments are calculated using the known input geometry.

\section{CONCLUSION}

First, this paper research a test bench for a kind of micro turbine engine. It can measurement exhaust temperature, vibration, static and dynamic pressure and thrust. Second, use the finite element analysis software analysis the natural frequency. At last give the method of thrust stand calibration procedure.

\section{REFERENCES}

[1] Pfister, M. G. Development of a Very Small Aero-Engine. In Proceedings of the ASME Turbo Expo 200045 th ASME International Gas Turbine and Aeroengine Technical Congress and Exposition: Munich, Germany, 2000

[2] J. Peirs, D.Reynaerts, F.Verplaetsen, etal. Development of a Micro Gas Turbine For Electric Power Generation. The 14TH Micro Mechanics Europe Workshop, 2003,2(4): 215 219

[3] Lin, H. D. C. A. C. H. L. A. R. An Investigation of Steady and Dynamic Performance of a Small Turbojet Engine. In Proceedings of ASME Turbo Expo 2002

[4] Hsiao-Wei,D,Chiang,Chih-Neng Hsu,Yi-Ming Huang. Dynamic Performance of a small Turbojet Engine, International Journal of Turbo and Engines, 2003,(20): 195 207

[5] HAAG T W. Thrust Stand for Pulsed Plasma Thrusters. Review of Scientific Instruments, 1997, 5(68):2060-2067

[6] http://www.elwingcorp.com/2012/10/nasa-marshall-space-flightcenter-to-test-eligos-inc-electrodeless-plasma-thruster-underspace-act-agreement/

[7] Liang dewang,Huang guoping. Main research progress of centimeter level micro turbojet engine. Gas turbine test and research. 2004, 17(2): 9〜13

[8] Ma xiangdong,Huang guoping,Zhang tianhong. MTE-110 The micro turbojet engine overall performance modeling and experimental research. The second academic year at the Chinese academy of space. 2006

[9] Spencer Sessions.A 6-Degree of Freedom Static Thrust Stand Developed for RC-Scale Jet Engines: [Master degree]. Utah: Utah State University, 2011

[10] M. Ryan Schaefermeyer, Stephen A. Whitmore, Cordell B. Wright. Maneuvering and Gravity Offset Flight Controls for an Extraterrestrial Surface Landing Research Vehicle. 46th AIAA/ASME/SAE/ASEE Joint Propulsion Conference \& Exhibit, 2010

[11] M. Ryan Schaefermeyer. Aerodynamic Thrust Vectoring For Attitude Control Of A Vertically Thrusting Jet Engine: [Master degree]. Utah: Utah State University, 2011

[12] Shannon D. Eilers, Matthew D. Wilson, Stephen A. Whitmore. Analytical and Experimental Evaluation of Aerodynamic Thrust Vectoring on an Aerospike Nozzle. 46th AIAA/ASME/SAE/ASEE Joint Propulsion Conference \& Exhibit, 2010 